BULLETIN OF THE

AMERICAN MATHEMATICAL SOCIETY

Volume 78, Number 4, July 1972

\title{
SOME RESULTS ON SEPARATING FUNCTION ALGEBRAS
}

\author{
BY G. L. CSORDAS AND H. B. REITER
}

Communicated by Felix Browder, December 29, 1971

1. Introduction. Recent results of Hoffman and Singer [7], Weiss [10], and Wilken [11] indicate that the study of separation properties play a central role in the theory of function algebras. Our purpose in this note is to announce some results concerning a natural separation property of function algebras. A more detailed exposition and the proofs of the results will be given elsewhere.

Let $X$ be a compact Hausdorff space and $C(X)$ the Banach algebra of all continuous complex-valued functions on $X$ with the usual supremum norm. Let $A$ be a function algebra on $X$. In the sequel we will call a closed subset $S$ of $X$ an $L_{A^{-}}$set or briefly an $L$-set, if $L(S)=S$, where

$$
L(S)=\bigcap_{f \in A} f^{-1} f(S) .
$$

We will say that an algebra $A$ on $X$ is a separating algebra if every closed subset of $X$ is an $L$-set. It is clear that regular, approximately normal, and approximately regular algebras (see Wilken [11] for the terminology) are all examples of separating algebras. The concept of a separating algebra is quite broad as the example of the disk algebra shows. Although the disk algebra is a separating algebra, it is neither maximal nor approximately regular.

On the other hand, it is quite often difficult to determine whether or not an algebra is a separating algebra. For instance, it is not known whether or not $H^{\infty}$ considered as an algebra of functions on its maximal ideal space is a separating algebra.

2. Properties of $L$-sets. $L$-sets abound in function algebras. Clearly, the whole space $X$, each singleton and any finite subset of $X$ are $L$-sets. Also hull-kernel closed sets, maximal sets of antisymmetry, the essential set of an algebra, and the arbitrary intersection of $L$-sets provide additional examples of $L$-sets. (For the terminology used here see Browder [2] and Gamelin [4].)

Direct verification yields the following:

LEMMA 2.1. If $S$ and $T$ are any two closed subsets of $X$, then (i) $L(S) \subseteq L(T)$, whenever $S \subseteq T$, and (ii) $L(S)=L(L(S)$ ).

We remark that in general $L$ is not a closure operator. For example,

AMS 1970 subject classifications. Primary 46J10, 46J20; Secondary 46J30, 54D15. 
consider the algebra $A$ of all functions continuous on the closed unit bidisk,

$$
P=\{(z, w)|| z|\leqq 1,| w \mid \leqq 1\},
$$

in complex two-space which are analytic on the interior of the bidisk. Now, if

$$
S=\{(z, w)|| z|\leqq 1,| w \mid=1\},
$$

and

$$
T=\{(z, w)|| z|=1,| w \mid \leqq 1\},
$$

then $L(S \cup T) \neq L(S) \cup L(T)$. Another example may be found in Rudin $[9$, Theorem 1].

The earlier examples demonstrate that the union of two $L$-sets need not be an $L$-set. However, if we impose the additional restriction that one of the sets be a finite set, then we have the following theorem.

THEOREM 2.2. Let $S$ be an $L$-set, then $L(S \cup T)=S \cup T$ for any finite subset $T$ of $X$.

The next theorem establishes the existence of minimal non- $L$-sets.

THEOREM 2.3. Let $S$ be a closed subset of $X$ and let $x_{0} \in L(S) \backslash S$. Then $X$ contains a minimal closed subset $T$ such that $x_{0} \in L(T) \backslash T$.

3. Separating algebras. The results of $\S 3$ imply that if $A$ is a separating algebra, then $L$ is a closure operator and $L$ defines a topology on $X$ which is equivalent to the given topology. This is the content of the following characterization of separating algebras.

THEOREM 3.1. A necessary and sufficient condition for an algebra $A$ on $X$ to be a separating algebra is that the union of any two $L$-sets be an $L$-set.

We recall that $A$ is a Dirichlet algebra on $X$ if the real parts of the functions in $A$ are uniformly dense in the real continuous functions on $X$.

THEOREM 3.2. Every Dirichlet algebra is a separating algebra.

In general, the converse of Theorem 3.2 is not true. For example, the disk algebra is a separating algebra but it is not a Dirichlet algebra.

Lemma 3.3. If $A$ is a maximal essential subalgebra of $C(X)$, then $A$ is a separating algebra.

The results of Bear [1] show that the study of function algebras can effectively be reduced to the study of essential algebras. Thus it is not surprising that we can weaken the hypothesis of Lemma 3.3 to obtain

THEOREM 3.4. If $A$ is a maximal subalgebra of $C(X)$, then $A$ is a separating algebra. 
In [7, p. 221] Hoffman and Singer gave an example of a function algebra which is pervasive but not maximal.

THEOREM 3.5. If $A$ is a pervasive subalgebra of $C(X)$, then $A$ is a separating algebra.

We pause for a moment to discuss the scope of the foregoing result. The proofs of the above results depend on the fact that, for function algebras which are maximal or pervasive, the Silov boundary, $\Gamma$, is the whole space $X$. We shall show now by means of an example that the condition $\Gamma=X$ is not sufficient to guarantee that all closed sets are $L$-sets. As before, let $P=\{(z, w)|| z|\leqq 1| w \mid, \leqq 1\}$ and let $T$ denote the topological boundary. Let $A$ be the bidisk algebra on $P$. Define $A_{0}$ by

$$
A_{0}=\left\{f \in C(P)|f| T \cup\{\theta\} \in A_{T \cup\{\theta\}}\right\}
$$

where $\theta=(0,0), f \mid T \cup\{\theta\}$ denotes the restriction of $f$ to $T \cup\{\theta\}$, and where $A_{T \cup\{\theta\}}$ denotes the closure of $A$ restricted to $T \cup\{\theta\}$. Then $A_{0}$ is not a separating algebra on the connected space $P$, and the Silov boundary of $A_{0}$ is all of $P$ since each point in the interior of $P \backslash\{\theta\}$ is a peak point of $A_{0}$.

We conclude this paper with two important unanswered questions.

(1) Let $M_{A}$ denote the maximal ideal space and $\Gamma_{A}$ the Silov boundary of the algebra $A$. Is there a nonseparating essential algebra $A$ on a (connected) space $X$ for which $M_{A}=\Gamma_{A}=X$ ?

(2) Let $H_{\alpha}^{\infty}$ denote the function algebra obtained by restricting $H^{\infty}$ to the fiber $M_{\alpha}$. (See Hoffman $[5$, p. 187] for the terminology.) Is every closed subset of the maximal ideal space $M_{\alpha}$ of $H_{\alpha}^{\infty}$ an $L$-set? In this connection we note that not every closed set $S$ in $M_{\alpha}$ is "generated" by a set in the open unit disk $D$ in the sense that it is equal to $\bar{T} \cap M_{\alpha}$ for some $T \subseteq D$. This was pointed out to us in a written communication from P. M. Gauthier.

\section{BIBLIOGRAPHY}

1. H. S. Bear, Complex function algebras, Trans. Amer. Math. Soc. 90 (1959), 383-393. MR 21 \# 5889.

2. A. Browder, Introduction to function algebras, Benjamin, New York, 1969, MR 39 \# 7431.

3. L. Carleson, Interpolations by bounded analytic functions and the corona problem, Ann. of Math. (2) 76 (1962), 547-559. MR 25 \# 5186.

4. T. W. Gamelin, Uniform algebras, Prentice-Hall, Englewood Cliffs, N.J., 1969.

5. K. Hoffman, Banach spaces of analytic functions, Prentice-Hall Series in Modern Analysis, Prentice-Hall, Englewood Cliffs, N.J., 1962. MR 24 \# A2844.

6. K. Hoffman and I. M. Singer, Maximal subalgebras of $C(\Gamma)$, Amer. J. Math. 79 (1957), 295-305. MR 19, 46.

7. MR 22 \# 8318.

8. L. Hörmander, An introduction to complex analysis in several variables, Van Nostrand, Princeton, N.J., 1966. MR 34 \# 2933.

9. W. Rudin, Subalgebras of spaces of continuous functions, Proc. Amer. Math. Soc. 7 (1956), 825-830. MR 18, 587. 
10. M. L. Weiss, Some separation properties in sup-norm algebras of continuous functions, Proc. Internat. Sympos. on Function Algebras (Tulane Univ., 1965), Scott-Foresman, Chicago, Ill., 1966, pp. 93-97. MR 33 \# 1756.

11. D. R. Wilken, Approximate normality and function algebras on the interval and the circle, Proc. Internat. Sympos. on Function Algebras (Tulane Univ., 1965), Scott-Foresman. Chicago, Ill., 1966, pp. 98-111. MR 33 \# 4712.

Department of Mathematics. University of Hawali, Honolulu, Hawall 96822 